\title{
MORSE THEORY ON KÄHLER HOMOGENEOUS SPACES ${ }^{1}$
}

\author{
GEORGE D. PARKER
}

\begin{abstract}
By exploiting the Kähler structure, we find a Morse function $f$ on the homogeneous space $\mathfrak{b} / C\left(\mathfrak{T}^{\prime}\right)$. The homology is readily computed and is seen to be contained in the diagram of $\mathfrak{G}$. The Morse cells are shown to be complex analytic submanifolds and to coincide with those of a cell decomposition found by Borel in a different manner.
\end{abstract}

In [1] Borel proved that the compact Kähler homogeneous spaces were precisely the coset spaces $\mathfrak{b} / \mathfrak{R}$, where $\mathfrak{G}$ is a compact semisimple Lie group and $\Re$ is the centralizer of a toral subgroup. He also gave the first proof that such spaces have torsion free homology with odd Betti numbers vanishing by demonstrating a complex analytic cell decomposition. Bott [3] then applied Morse theory via his theory of variational completeness and actually computed the Betti numbers, showing how they may be read from the diagram of $(5$.

Below we shall find a Morse function via a different method, completing a program of $\mathrm{T}$. Frankel. The results will combine both of the above: the Betti numbers will be found via the diagram and the Morse cells will turn out to be the same ones that Borel found and hence will be complex analytic submanifolds.

We assume the reader has some knowledge of Lie theory, roots, diagram, and the like.

1. The basic method. We apply the following result of Frankel, whose proof may be found in [5]. Let $M$ be a compact Kähler manifold with Kähler form $\Omega$ and let $\left\{\varphi_{t}\right\}$ be a one-parameter group of isometries of $M$ with Killing field (infinitesimal isometry) $\tilde{X}$ and fixed set $F$.

Received by the editors October 15, 1971.

AMS 1970 subject classifications. Primary 57D70, 57F15, Secondary 57E25.

Key words and phrases. Morse theory, Kähler manifold, homogeneous space, Killing field, Morse-Bott inequalities, infinitesimal diagram of $\mathfrak{b}$, cell decomposition.

${ }^{1}$ Research supported in part by an NSF Graduate Fellowship and NSF Grant GP20863. 
THEOREM. If either $b_{1}(M ; R)=0$ or $F \neq \varnothing$, then $\iota_{\tilde{X}} \Omega$ (interior product by $\tilde{X})=-d f$, where $f$ is a smooth function with $F$ as critical set. The critical points form nondegenerate critical manifolds in the sense of Bott [4], the Morse indices are all even, and the Morse-Bott inequalities are equalities for all coefficient fields: $b_{j}(M)=\sum_{k} b_{j-\lambda_{k}}\left(F_{k}\right)$, where the $F_{k}$ are the components of $F$ and $\lambda_{k}$ is the Morse index of $f$ on $F_{k}$.

Frankel's proof indicates the following way to compute the indices. If $x \in F_{k}, \varphi_{t^{*}}$ rotates $T M_{x}$, the tangent space to $M$ at $x$. $T M_{x}$ splits as $T M_{x}=$ $T\left(F_{k}\right)_{x} \oplus \oplus e_{r}$, where each $e_{r}$ is two dimensional and invariant under both $\varphi_{t^{*}}$ and the almost complex structure operator $J$. The index at $x$ equals twice the number of those $e_{r}$ which $\varphi_{t^{*}}$ rotates negatively with respect to the orientation of $J$.

2. Notation and preliminaries. Let $\mathfrak{5}$ be a compact semisimple Lie group. We shall assume that $\mathfrak{5}$ is centerless and hence equal to its own adjoint group without loss of generality below. Let $\mathfrak{I}^{\prime}$ be a toral subgroup of $\left(\mathfrak{5}, \mathfrak{R}=C\left(\mathfrak{I}^{\prime}\right)\right.$, and $\mathfrak{I}$ a maximal torus of $\left(\mathfrak{5}\right.$ containing $\mathfrak{I}^{\prime}$. Denote the coset $g \Re$ by $\bar{g}$.

Let $Y$ be tangent to $\mathfrak{I}^{\prime}$ at the identity $e$ such that $|\alpha(Y)|<\frac{1}{2}$ for all roots $\alpha$ of $\left(\mathfrak{5}\right.$ and such that $\exp (t Y)$ is a dense one-parameter subgroup of $\mathfrak{I}^{\prime}$. Choose a Weyl chamber $\mathscr{F}$ for $(5$ that contains $Y$ in its closure. The roots of $\mathfrak{F}$ that are positive in $\mathscr{F}$ are positive for some lexicographic ordering of the roots and shall be denoted as a set by $\Sigma$.

The Lie algebra $\mathfrak{g}$ of $\mathfrak{b}$ splits under the adjoint action of $\mathfrak{I}$ as $\mathfrak{g}=\mathfrak{t} \oplus \oplus \mathfrak{a}_{\alpha}$, where each $a_{\alpha}$ is two dimensional and $\operatorname{Ad}(\exp S)$ acts on $\mathfrak{a}_{\alpha}$ by rotation through an angle $2 \pi \alpha(S)$.

If $\Sigma^{\prime}$ denotes the set of positive roots not vanishing identically on $\mathfrak{T}_{e}^{\prime}$, then $(\mathfrak{S} / \mathfrak{R})_{\bar{e}} \simeq \sum_{\Sigma^{\prime}} \mathfrak{a}_{\alpha}$. One defines a complex structure on each $\mathfrak{a}_{\alpha}, \alpha \in \Sigma^{\prime}$, whose orientation agrees with that induced by $\alpha$ and which is invariant under the adjoint action of $\Re$ (see [2, pp. 497-498] for details). $J$ extends via left translation to a homogeneous almost complex structure on $\mathbb{5} / \Re$. According to a theorem of Frölicher [6, pp. 91-92], $J$ is integrable and hence gives a complex structure. This complex structure is the same as the natural one that arises from the following.

$\boldsymbol{C} \otimes \mathfrak{a}_{\alpha} \subset \boldsymbol{C} \otimes \mathfrak{g}=\mathfrak{g}^{\boldsymbol{C}}$ is spanned by two complex vectors $E_{\alpha}$ and $E_{-\alpha}$ where $E_{\alpha}+E_{-\alpha}$ and $i\left(E_{\alpha}-E_{-\alpha}\right)$ span $\mathfrak{a}_{\alpha}$ in the standard way. Let $\mathrm{I}_{Y}$ be the subalgebra of $\mathrm{g}^{C}$ spanned by $\boldsymbol{C} \otimes \mathrm{t}=\mathrm{t}^{C},\left\{E_{\alpha} \mid \alpha \in \Sigma\right\}$, and $\left\{E_{-\alpha} \mid \alpha(Y)=0\right\}$. Then if $\mathfrak{L}_{Y}$ is the adjoint group of $\mathfrak{I}_{Y}$, viewing $\mathfrak{L}_{Y} \subset \mathfrak{5}^{C}=$ adjoint group of $\mathrm{g}^{C}$, $\mathfrak{G}^{C} / \mathfrak{Q}_{Y} \simeq \mathfrak{b} / \mathfrak{R}$, the isomorphism being induced by the inclusion $\mathfrak{G} \subset \mathfrak{F}^{C}$. Using left invariant Maurer-Cartan forms on $\mathfrak{5}^{C}$ dual to the $E_{\alpha}$, one can create a Kähler form on $\mathfrak{G}^{C} / \mathfrak{Q}_{Y}$ whose complex structure is the natural one induced from that of $\mathfrak{5}^{C}[\mathbf{1}]$. 
3. Morse theory on $\mathfrak{G} / \mathfrak{R}$. Let $X \in \mathscr{F}$ so that $\exp (t X)$ gives a dense oneparameter subgroup of $\mathfrak{I}$. $\exp (t X)$ acts on $\mathfrak{F} / \mathfrak{R}$ by $\bar{g} \mapsto(\exp (t X) g)^{-}$, the action being isometric since the Kähler structure is homogeneous.

Lemma. The fixed set of the action is $\{\bar{g} \mid g \in N(\mathfrak{I})=$ normalizer of $\mathfrak{I}\}$. The fixed points are in one-to-one correspondence with the left cosets of $W_{\mathfrak{R}}$ in $W_{\mathfrak{G}}$, where $W_{\mathfrak{G}}=N(\mathfrak{I}) / \mathfrak{I}$ and $W_{\mathfrak{R}}=(\mathfrak{R} \cap N(\mathfrak{I})) / \mathfrak{I}$ are the Weyl groups of $\mathfrak{G}$ and $\mathfrak{R}$.

Proof. $\quad \bar{g}$ is fixed iff $\bar{g}=(\exp (t X) g)^{-}$for all $t$, iff $g^{-1} \exp (t X) g \in \mathfrak{R}$ for all $t$, iff $g^{-1} \mathfrak{I} g \subset \Re$. Since both $g^{-1} \mathfrak{I} g$ and $\mathfrak{I}$ are maximal tori of $\Re$, they are conjugate in $\mathfrak{R}$ and there exists $h \in \mathfrak{R}$ so that $g^{-1} \mathfrak{I} g=h \mathfrak{T} h^{-1}$. Then $g h \mathfrak{I}(g h)^{-1}=\mathfrak{I}$ and $g h \in N(\mathfrak{I})$. Since $\bar{g}=(g h)^{-}$, the first assertion follows.

If $g, h \in N(\mathfrak{I})$, then $\bar{g}=\bar{h}$ iff $g^{-1} h \in \mathfrak{R}$, iff $g^{-1} h \mathfrak{I} \in W_{\mathfrak{R}}$, iff $g \mathfrak{I} \equiv h \mathfrak{T}$ $\left(\bmod W_{\Re}\right)$. Hence we have the second assertion.

Thus there are precisely $\left|W_{\mathfrak{G}} / W_{\mathfrak{R}}\right|$ fixed points and, as was pointed out by Frankel [5, Corollary 3], since the indices are all even, $\mathfrak{F} / \mathfrak{R}$ has torsion free homology with the odd Betti numbers vanishing.

To compute the index of Frankel's function $f$ at a critical point $\bar{g}$ with $g \in N(\mathfrak{I})$, we need to know how $L_{\exp (t X)^{*}}$ acts on $(\mathfrak{G} / \mathfrak{R})_{\bar{g}}$. Now

$$
(\mathfrak{G} / \mathfrak{R})_{\bar{g}} \stackrel{L_{\left(g^{-1}\right)^{*}}}{\simeq}(\mathfrak{G} / \mathfrak{R})_{\bar{e}} \simeq \sum_{\Sigma^{\prime}} \mathfrak{a}_{\alpha}
$$

$L_{\exp (t X) *}$ lifts then to $L_{\left(g^{-1}\right) *} \circ L_{\exp (t X) *} \circ L_{g *}=L_{\left(g^{-1} \exp (t X) g\right) *}$ on $\left((\mathfrak{5} / \Re)_{\bar{e}}\right.$ and to $\operatorname{Ad}\left(g^{-1} \exp (t X) g\right)$ on $\sum_{\Sigma^{\prime}} \mathfrak{a}_{\alpha}$.

If $g \in N(\mathfrak{I})$, denote $\left.\operatorname{Ad}(g)\right|_{\mathfrak{I}_{e}}$ by $\varphi_{g}\left(\varphi_{g} \in W_{\mathfrak{G}}\right)$. Then $L_{\exp (t X) *}$ lifts to $\oplus R\left(2 \pi \alpha\left(\varphi_{g}^{-1}(X)\right)\right)$ on $\sum_{\Sigma^{\prime}} \mathfrak{a}_{\alpha}$. The rotation is negative on the two-plane $\mathfrak{a}_{\alpha}$ with respect to the orientation of $J$ iff $\alpha\left(\varphi_{g}^{-1}(X)\right)<0$, iff $X$ and $\varphi_{g}^{-1}(X)$ lie on opposite sides of the plane $\alpha=0$ in the diagram of $\mathfrak{b}$.

Thus the index at $\bar{g}$ is $2 \#\left\{\alpha \in \Sigma^{\prime} \mid\right.$ line segment from $X$ to $\varphi_{g}^{-1}(X)$ crosses the plane $\alpha=0\}=2 \#\left\{\alpha \in \Sigma^{\prime} \mid\right.$ line from $Y$ to $\varphi_{g}^{-1}(X)$ crosses $\left.\alpha=0\right\}=$ $2 \#\{$ planes of the infinitesimal diagram of $(5$ crossed by the open line segment from $Y$ to $\left.\varphi_{g}^{-1}(X)\right\} \equiv 2 X_{Y}^{*}\left(\varphi_{g}\right)$. (Note that Bott's counting function $X^{*}(\varphi)$ is not well defined unless $\mathfrak{I}^{\prime}=\mathfrak{I}[1$, p. 252].) Hence the index may be read from the diagram of $(5$ and we have the following.

TheOREM. The Poincaré polynomial of $\mathfrak{G} / \mathfrak{R}$ for any coefficient field is

$$
P(\mathfrak{S} / \Re ; t)=\sum_{\begin{array}{c}
\text { coset reps. } \\
\text { of } W_{\mathfrak{R}} \text { in } W_{\mathfrak{G}}
\end{array}} t^{2\left(X_{Y}^{*}(\varphi)\right)}=\frac{1}{\left|W_{\mathfrak{R}}\right|} \sum_{W_{\mathfrak{G}}} t^{2\left(X_{Y}^{*}(\varphi)\right)} \text {. }
$$


4. The cell structure. Recall that the Morse cell (or "bowl", in Morse's terminology) about an isolated critical point $p, M C(p)$, is the union of $p$ with all open gradient lines (having the sense of increasing $f$ ) that terminate at $p$. The Morse cell at $p$ is diffeomorphic to an open Euclidean $\lambda$-cell, where $\lambda$ is the index of $f$ at $p$, and has as tangent space at $p$ the subspace of $T M_{p}$ spanned by the eigenvectors of the Hessian linear transformation whose eigenvalues are negative. If one has a Morse function with isolated critical points, the Morse cells give a cell decomposition of $M$.

Now Borel found a cell decomposition of $\mathfrak{5} / \mathfrak{R}$ in the following way. Let $\mathfrak{L} \subset \mathfrak{G}^{C}$ be the adjoint group of $\mathfrak{l}=\mathrm{t}^{C} \oplus \oplus_{\Sigma} C E_{\alpha} \cdot \mathfrak{L}$ acts on $\left(\mathfrak{F}^{C} / \mathfrak{L}_{Y} \simeq \mathfrak{F} / \mathfrak{R}\right.$ complex analytically on the left. Because the action is complex, the orbits are complex submanifolds and of even dimension. Borel asserted that these orbits are birationally and biregularly equivalent to complex affine spaces. We claim the following.

THEOREM. The orbits under $\mathfrak{L}$ coincide with the Morse cells off. Hence the Morse cells are complex analytic submanifolds and the orbits are cells.

Proof. Since $\exp (t X) \in \mathfrak{I} \subset \mathfrak{Q}$, the orbits under $\mathfrak{L}$ are invariant under the one-parameter group of isometries. Thus $\tilde{X}$ (=Killing field) is tangent to the orbits and so is $J \tilde{X}$. Since $-d f=\iota_{\tilde{X}} \Omega$ and $\Omega(\cdot, \cdot) \equiv g(J \cdot, \cdot)$, where $g$ is the Riemannian metric, $J \tilde{X}=-\operatorname{grad} f$. Thus each orbit is a union of gradient lines and critical points.

Harish-Chandra has shown [7] that $\mathfrak{L} g \mathfrak{Q} \cap N(\mathfrak{I}) \neq \varnothing$ for any $g \in \mathfrak{G}^{\boldsymbol{C}}$, hence $\mathfrak{L} g \mathfrak{L}_{Y} \cap N(\mathfrak{I}) \neq \varnothing$ and each orbit contains at least one critical point. One may show directly that each orbit contains at most one critical point or one can use the following counting argument. By Borel's theorem, the total Betti number equals the number of Borel cells. But by Frankel's theorem the total Betti number equals the number of critical points. Since each orbit contains at least one critical point, it must contain exactly one.

Let $\bar{g}$ be a critical point, $g \in N(\mathfrak{I})$.

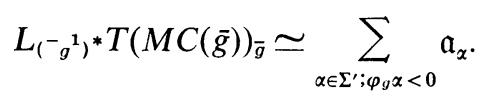

Let $\alpha \in \Sigma^{\prime}$ with $\varphi_{g} \alpha<0$. Now $E_{\alpha}$ is tangent to $\mathfrak{I}_{Y}$ at $e$ and thus to $g^{-1} \mathfrak{Q} g \mathfrak{L}_{Y}$. Since $\varphi_{g} \alpha<0, E_{-\varphi_{g} \alpha}$ is tangent to $\mathfrak{L}$ at $e$ and $\operatorname{Ad}\left(g^{-1}\right) E_{-\varphi_{g} \alpha}=\mu E_{-\alpha}(\mu \neq 0)$ is tangent to $g^{-1} \mathfrak{Q} g$ and thus to $g^{-1} \mathfrak{Q} g \mathfrak{L}_{Y}$ at $e$. Thus both $\left(E_{\alpha}+E_{-\alpha}\right)$ and $i\left(E_{\alpha}-E_{-\alpha}\right)$ are tangent to $g^{-1} \mathfrak{Q} g \mathfrak{Q}_{Y} \cap \mathfrak{G}$. Hence under the projection $\mathfrak{G} \rightarrow \mathfrak{G} / \mathfrak{R}$ and the isomorphism $\sum_{\alpha \in \Sigma^{\prime}} \mathfrak{a}_{\alpha} \simeq T(\mathfrak{G} / \mathfrak{R})_{\bar{e}}, \quad\left(E_{\alpha}+E_{-\alpha}\right)$ and $i\left(E_{\alpha}-E_{-\alpha}\right)$ are both tangent to $L_{(g-1)} B C(\bar{g})$ at $e$. Hence $T(M C(\bar{g}))_{\bar{g}} \subset$ $T(B C(\bar{g}))_{\bar{g}}$ and $M C(\bar{g}) \subset B C(\bar{g})$ since both are unions of gradient lines having $\bar{g}$ as an end point. Since both sets of cells partition $(\mathfrak{G} / \Re$, we must have $M C(\bar{g})=B C(\bar{g})$. 
5. A conjecture. Since the Morse cells in the previous case turned out to be complex analytic submanifolds, it is reasonable to pose the following.

CONJECTURE. Let $M$ be a compact Kähler manifold on which a oneparameter group acts by isometries with isolated fixed points. Then the Morse cells arising from Frankel's function are complex analytic submanifolds.

At present, besides the above case, the author has as examples of this the complex Grassmannians (which have the form $\mathfrak{G} / \mathfrak{R}$, but a different action is used and the Morse cells turn out to be the Schubert cells) and some contrived examples formed by blowing up points on projective spaces and the like.

\section{BIBLIOGRAPHY}

1. A. Borel, Kählerian coset spaces of semisimple Lie groups, Proc. Nat. Acad. Sci. U.S.A. 40 (1954), 1147-1151. MR 17, 1108.

2. A. Borel and F. Hirzebruch, Characteristic classes and homogeneous spaces. I, Amer. J. Math. 80 (1958), 458-538. MR 21 \#1586.

3. R. Bott, An application of the Morse theory to the topology of Lie groups, Bull. Soc. Math. France 84 (1956), 251-281. MR 19, 291.

4. - Nondegenerate critical manifolds, Ann. of Math. (2), 60 (1954), 248-261. MR 16, 276.

5. T. Frankel, Fixed points and torsion on Kähler manifolds, Ann. of Math. (2) 70 (1959), 1-8. MR 24 \#A1730.

6. A. Frölicher, Zur Differentialgeometrie der complexen Strukturen, Math. Ann. 129 (1955), 50-95. MR 16, 857.

7. Harish-Chandra, On a lemma of F. Bruhât, J. Math. Pures Appl. (9) 35 (1956), 203-210. MR 18, 137.

Department of Mathematics, University of California at San Diego, La Jolla, CALIFORNIA 92037

Current address: Department of Mathematics, Southern Illinois University, Carbondale, Illinois 62901 